\title{
Correspondence
}

\section{Streaming potential effects on solute dispersion in}

\section{nanochannels}

\author{
Xiangchun Xuan \\ Department of Mechanical Engineering, Clemson University, Clemson, SC 29634, USA \\ E-mail: xcxuan@clemson.edu. Tel: (864) 656-5630. Fax: (864) 656-7299.
}

This Supporting Information includes the derivation of the pressure-driven flow induced streaming potential field, eq 12, and the derivation of the electrokinetic "figure of merit", eq 15 .

Combining equations 2 and 11 yields the electric current density $j$ in electrokinetic flow along a slit channel,

$$
j=-\frac{\varepsilon R T}{2 \mu F} \frac{d^{2} \Psi}{d y^{2}}\left(1-y^{2}\right) P+\frac{\varepsilon^{2} \zeta R T}{a^{2} \mu F} \frac{d^{2} \Psi}{d y^{2}}\left(1-\frac{\Psi}{\zeta^{*}}\right) E+c_{b} \lambda_{b} \cosh (\Psi) E
$$

The zero electric current in a steady-state pressure-driven flow forces

$$
0=-\frac{\varepsilon R T}{2 \mu F} \int_{0}^{1} \frac{d^{2} \Psi}{d y^{2}}\left(1-y^{2}\right) d y P+\frac{\varepsilon^{2} \zeta R T}{a^{2} \mu F} \int_{0}^{1} \frac{d^{2} \Psi}{d y^{2}}\left(1-\frac{\Psi}{\zeta^{*}}\right) d y E_{s t}+c_{b} \lambda_{b} \int_{0}^{1} \cosh (\Psi) d y E_{s t} .(\mathrm{S} 2)
$$

Therefore, the induced streaming potential field is determined as 


$$
E_{s t}=\frac{\frac{\varepsilon R T}{2 \mu F} \int_{0}^{1} \frac{d^{2} \Psi}{d y^{2}}\left(1-y^{2}\right) d y}{\frac{\varepsilon^{2} \zeta R T}{a^{2} \mu F} \int_{0}^{1} \frac{d^{2} \Psi}{d y^{2}}\left(1-\frac{\Psi}{\zeta^{*}}\right) d y+c_{b} \lambda_{b} \int_{0}^{1} \cosh (\Psi) d y} P
$$

Employing the integration by parts rewrites eq $\mathrm{S} 3$ as

$$
E_{s t}=\frac{\frac{\varepsilon R T \zeta^{*}}{\mu F} g_{1}}{\frac{\varepsilon^{2} R^{2} T^{2}}{a^{2} \mu F^{2}} \int_{0}^{1}\left(\frac{d \Psi}{d y}\right)^{2} d y+c_{b} \lambda_{b} g_{3}} P
$$

where the functions $g_{1}$ and $g_{3}$ are as defined in the main text. Multiplying the two sides of the Poisson-Boltzmann equation by $2 d \Psi / d y$ and then integrating the new eq 3 leads to

$$
\left(\frac{d \Psi}{d y}\right)^{2}=2 \kappa^{* 2}\left[g_{3}-\cosh \left(\Psi_{0}\right)\right] .
$$

Thus, eq S4 can be simplified to eq 12 after mathematical manipulations, wherein the expression $\kappa^{* 2}=2 a^{2} F^{2} c_{b} / \varepsilon R T$ has been invoked.

As for the definition of electrokinetic "figure of merit", we first get the following relationship by area-averaging the two fluid velocity components in eq 2

$\frac{\left\langle u_{e}\right\rangle}{\left\langle u_{p}\right\rangle}=-\frac{3 \varepsilon \zeta}{a^{2}} g_{1} \frac{E_{s t}}{P}$.

Then, replacing $E_{s t}$ with eq 12 and invoking $\kappa^{* 2}=2 a^{2} F^{2} c_{b} / \varepsilon R T$ again receive

$$
\frac{\left\langle u_{e}\right\rangle}{\left\langle u_{p}\right\rangle}=-\frac{3 \zeta^{* 2} g_{1}^{2}}{2 \phi^{2}\left[(1+\beta / 4) g_{3}-\cosh \left(\Psi_{0}\right)\right]}=-Z
$$

